\title{
Radiogenic Source Identification for the Helium Production-Diffusion Equation
}

\author{
Gang $\mathrm{Bao}^{1}$, Todd A. Ehlers ${ }^{2}$ and Peijun $\mathrm{Li}^{3, *}$ \\ ${ }^{1}$ Department of Mathematics, Zhejiang University, Hangzhou 310027, China; \\ Department of Mathematics, Michigan State University, East Lansing, MI 48824, \\ USA. \\ 2 Department of Geosciences, Wilhelmstrasse 56, Universität Tübingen, D-72074, \\ Tübingen, Germany. \\ ${ }^{3}$ Department of Mathematics, Purdue University, West Lafayette, IN 47907, USA.
}

Received 3 January 2012; Accepted (in revised version) 25 May 2012

Available online 18 October 2012

\begin{abstract}
Knowledge of helium diffusion kinetics is critical for materials in which helium measurements are made, particulary for thermochronology. In most cases the helium ages were younger than expected, an observation attributes to diffusive loss of helium and the ejection of high energy alpha particles. Therefore it is important to accurately calculate the distribution of the source term within a sample. In this paper, the prediction of the helium concentrations as function of a spatially variable source term are considered. Both the forward and inverse solutions are presented. Under the assumption of radially symmetric geometry, an analytical solution is deduced based on the eigenfunction expansion. Two regularization methods, the Tikhonov regularization and the spectral cutoff regularization, are considered to obtain the regularized solution. Error estimates with optimal convergence order are shown between the exact solution and the regularized solution. Numerical examples are presented to illustrate the validity and effectiveness of the proposed methods.
\end{abstract}

AMS subject classifications: 65M32, 35Q80

Key words: Inverse source problem, production-diffusion equation, Tikhonov regularization.

\section{Introduction}

Helium isotopes are used extensively as thermochronometer in terrestrial and extraterrestrial materials [20]. He produced from radioactive decay of uranium and thorium

${ }^{*}$ Corresponding author. Email addresses: bao@math.msu.edu (G. Bao), todd.ehlers@uni-tuebingen.de (T. A. Ehlers), lipeijun@math.purdue.edu (P. Li) 
series nuclides forms the basis of (U-Th)/He chronometry. The main attraction of helium for these applications is that its production rates are high compared to other isotope systems, coupled with the fact that high-precision, high-sensitivity helium analysis are comparatively easy. A critical consideration for these uses is that helium diffusion in most minerals occurs at moderate temperatures; failure to consider diffusive loss can lead to erroneously young helium-based age constraints. Knowledge of helium diffusion kinetics is therefore critical for materials in which helium measurements are made, particulary for thermochronology.

Despite of the appeal of (U-Th)/He system for thermochronological studies, one drawback of the technique is that spatial variations in radiogenic uranium and thorium in a sample can cause a non-uniform production of helium, and violate commonly made assumptions of a uniform source [9]. Spatial variations in uranium and thorium (often referred to a zoning of the parent isotopes) can produce substantial spatial fractionation of the parent/daughter ratio in accessory minerals likely to be used for helium thermochronometry. In the experience of apatite, zircon, titanite are commonly zoned and can limit the ultimate precision of helium ages [23]. Therefore it is important to accurately know the distribution of parent uranium and thorium isotopes (the source function) in a sample to be of use in helium dating. In this paper, the prediction of the a variable source term is formulated as an inverse radiogenic source problem.

Our model of helium production and diffusion considers the local helium concentration gradients resulting from ejection of high energy alpha particles from grain surfaces. It is assumed that the grain is of a spherical diffusion geometry, which is actually consistent with laboratory measurements of helium diffusion from apatite [22]. As a consequence of radiogenic production and diffusive loss, the concentration of helium as a function of the dimensional radial variable $\rho$ within the spherical diffusion domain of radius $R$ is [20]:

$$
\frac{\partial u(t, \rho)}{\partial t}=a(t)\left[\frac{\partial^{2} u(t, \rho)}{\partial \rho^{2}}+\frac{2}{\rho} \frac{\partial u(t, \rho)}{\partial \rho}\right]+f(\rho), \quad 0<t<T, \quad 0<\rho<R,
$$

where $a(t)$ is the time dependent diffusion coefficient, which is assumed to have a lower bound $a_{0}$ and an upper bound $a_{1}$, i.e.,

$$
0<a_{0} \leq a(t) \leq a_{1},
$$

and $f(\rho) \geq 0$ corresponds to the spatial variable dependent radiogenic source production. The homogeneous initial condition is prescribed

$$
u(0, \rho)=0, \quad 0<\rho<R .
$$

The boundary conditions are given by

$$
\lim _{\rho \rightarrow 0} u(t, \rho) \text { bounded, } \quad u(t, R)=0, \quad 0<t<T .
$$


The data is given as the final observation of the helium concentration:

$$
u(T, \rho)=g(\rho), \quad 0<\rho<R .
$$

Given the data function $g(\rho)$, the inverse radiogenic source problem is to reconstruct the source term $f(\rho)$, which accounts for the ejection of high energy alpha particles.

Besides the motivation of the radiogenic source identification for the helium diffusion, the inverse source identification problem for the parabolic equation has many other important applications, such as migration of groundwater, identification and control of pollution source and environmental protection. As a parameter identification type of problem, the inverse source problem has received much attention of both mathematical and numerical studies. We refer to Bushuyev [2], Cannon and Pérez-Esteva [4], Choulli and Yamamoto [6], Hettlich and Rundell, [10], Sakamoto and Yamamoto [19], and references cited therein for the discussions on the existence, uniqueness, and stability of the solution. Numerical methods may be found in Cheng et al. [5], Farcas and Lesnic [8], Johansson and Lesnic [14], Xie and Zou [24], Yamamoto and Zou [25], Yan et al. [26], and Yi and Murio [27,28]. One may consult Isakov [11,12] for a comprehensive discussion on the theoretical aspects of the inverse source problem, and Isakov [13] for accounts of general inverse problems for partial differential equations.

The goal of this work is to investigate mathematically and numerically the illposedness nature of the inverse radiogenic source problem. Under the assumption of a spherical diffusion geometry, the solution is explicitly constructed as an infinite series in terms of the eigenfunctions. Due to the rapidly decaying eigenvalues, small perturbation of the data can lead to large error of the reconstructed source function, which clearly shows the ill-posed nature of the problem. We consider two regularization methods, i.e., the Tikhonov regularization and the spectral cut-off regularization, in order to obtain a stable solution. Error estimates with optimal convergence are shown between the exact solution and the regularized solution. Numerical examples are presented to illustrate the validity and effectiveness of the proposed methods. This is part of a long-term research effort to quantifying tectonic and geomorphic interpretations of thermochronometer data with inverse problem theory. In the spirit of the collaborations in mathematics and geosciences, this interdisciplinary project targets questions and hypotheses that are fundamental to quantifying the evolution of mountain topography including the identification of the radiogenic source for the Helium production-diffusion equation. We refer to Bao et al. [1] for a related project on the reconstruction of mountain surfaces by quantifying tectonic and geomorphic interpretations of thermochronometer data.

The outline of this paper is as follows. Section 2 is devoted to the problem formulation and derivation of the solution in terms of the eigenfunction expansion. In Section 3, the regularization techniques are introduced to obtain stable solutions, and stability results are provided to the regularized solutions with explicit error estimates. Results of three numerical examples are presented in Section 4 . The paper is concluded with some general remarks and directions for future research in Section 5. 


\section{Problem formulation}

In this section, we derive an analytical solution for the inverse source problem based on the eigenfunction expansion. Throughout this paper, we denote by $L^{2}([0, R], \rho)$ the Hilbert space of Lebesgue measurable functions $u(\rho)$ with weight $\rho$ on $[0, R]$. Denote by $\langle\cdot, \cdot\rangle_{\rho}$ and $\|\cdot\|_{\rho}$ the inner product and norm on $L^{2}([0, R], \rho)$, respectively, given by

$$
\langle u, v\rangle_{\rho}=\int_{0}^{R} \rho u(\rho) v(\rho) d \rho \quad \text { and } \quad\|u\|_{\rho}=\left[\int_{0}^{R} \rho|u(\rho)|^{2} d \rho\right]^{1 / 2} .
$$

To apply the method of eigenfunction expansion for the function, we introduce a new function $v$ under the substitution

$$
u(t, \rho)=\rho^{-1 / 2} v(t, \rho) .
$$

It follows from the helium production-diffusion equation (1.1) that $v$ satisfies

$$
\frac{\partial v(t, \rho)}{\partial t}=a(t)\left[\frac{\partial^{2} v(t, \rho)}{\partial \rho^{2}}+\frac{1}{\rho} \frac{\partial v(t, \rho)}{\partial \rho}-\frac{1}{4 \rho^{2}} v(t, \rho)\right]+\rho^{1 / 2} f(\rho) .
$$

The corresponding initial condition is

$$
v(0, \rho)=0, \quad 0<\rho<R,
$$

the boundary conditions reduce to

$$
\lim _{\rho \rightarrow 0} \rho^{-1 / 2} v(t, \rho) \text { bounded, } \quad v(t, R)=0, \quad 0<t<T,
$$

and the final observation of the helium concentration becomes

$$
v(T, \rho)=\rho^{1 / 2} g(\rho), \quad 0<\rho<R .
$$

Applying the method of separation of variables, we seek a solution of (2.1) with the form

$$
v(t, \rho)=x(t) y(\rho) .
$$

Substituting (2.5) into (2.1), we require that $y(\rho)$ satisfies the equation:

$$
y^{\prime \prime}(\rho)+\frac{1}{\rho} y(\rho)+\left(\lambda-\frac{1}{4 \rho^{2}}\right) y(\rho)=0, \quad 0<\rho<R,
$$

and the boundary conditions:

$$
y(R)=0, \quad \lim _{\rho \rightarrow 0} \rho^{-1 / 2} y(\rho) \text { bounded, }
$$

where $\lambda$ is an unknown constant. 
Before proceeding to derive the eigenfunction expansion of the solution, we recall the following two Bessel functions of fraction orders [21]:

$$
J_{1 / 2}(z)=\left(\frac{2}{\pi z}\right)^{1 / 2} \sin z
$$

and

$$
J_{3 / 2}(z)=\left(\frac{2}{\pi z}\right)^{1 / 2}\left(\frac{\sin z}{z}-\cos z\right) .
$$

Clearly, the roots of the Bessel function $J_{1 / 2}(z)$ are:

$$
\mu_{n}=n \pi, \quad n=1,2, \cdots .
$$

According to [21], the eigenvalues of (2.6) are

$$
\lambda_{n}=\left(\frac{\mu_{n}}{R}\right)^{2}, \quad n=1,2, \cdots,
$$

and the corresponding eigenfunctions are

$$
y_{n}(\rho)=J_{1 / 2}\left(\frac{\mu_{n} \rho}{R}\right), \quad n=1,2, \cdots
$$

It follows from the properties of the Bessel function $J_{1 / 2}(z)$ that the eigenfunction system $y_{n}(\rho)$ are complete and orthogonal with weight $\rho$ in $L^{2}([0, R], \rho)$. Thus the solution $v(t, \rho)$ and the source term $\rho^{1 / 2} f(\rho)$ can be represented as

$$
\begin{aligned}
& v(t, \rho)=\sum_{n=1}^{\infty} x_{n}(t) y_{n}(\rho), \\
& \rho^{1 / 2} f(\rho)=\sum_{n=1}^{\infty} f_{n} y_{n}(\rho),
\end{aligned}
$$

where

$$
f_{n}=\frac{2}{R^{2} J_{3 / 2}^{2}\left(\mu_{n}\right)} \int_{0}^{R} \rho^{3 / 2} f(\rho) y_{n}(\rho) d \rho, \quad n=1,2, \cdots .
$$

Substituting (2.7) and (2.8) into (2.1), we have

$$
\begin{aligned}
& x_{n}^{\prime}(t)+\lambda_{n} a(t) x_{n}(t)=f_{n} \\
& x_{n}(0)=0 .
\end{aligned}
$$

Solving the above initial-value problem yields the solution

$$
x_{n}(t)=f_{n} \int_{0}^{t} \exp \left[-\lambda_{n} \int_{\tau}^{t} a(s) d s\right] d \tau
$$


Therefore the solution of (2.1) can be written as the infinite series

$$
v(t, \rho)=\sum_{n=1}^{\infty} f_{n} y_{n}(\rho) \int_{0}^{t} \exp \left[-\lambda_{n} \int_{\tau}^{t} a(s) d s\right] d \tau .
$$

Evaluating (2.9) at $t=T$ on both sides and using the final helium concentration (2.4) give

$$
\rho^{1 / 2} g(\rho)=\sum_{n=1}^{\infty} \kappa_{n} f_{n} y_{n}(\rho)
$$

where

$$
\kappa_{n}=\int_{0}^{T} \exp \left[-\lambda_{n} \int_{\tau}^{T} a(s) d s\right] d \tau
$$

Define

$$
w_{n}(\rho)=\frac{\sqrt{2}}{R J_{3 / 2}\left(\mu_{n}\right)} y_{n}(\rho) .
$$

It is easy to check that the eigenfunctions $w_{1}(\rho), w_{2}(\rho), \cdots, w_{n}(\rho), \cdots$ form an orthonormal basis in $L^{2}([0, R], \rho)$. Using the eigenfunctions as a basis, we have the representation of the data:

$$
\rho^{1 / 2} g(\rho)=\sum_{n=1}^{\infty} \kappa_{n}\left\langle\rho^{1 / 2} f(\rho), w_{n}(\rho)\right\rangle_{\rho} w_{n}(\rho) .
$$

It follows from the eigenfunction expansion (2.10) that

$$
\left\langle\rho^{1 / 2} g(\rho), w_{n}(\rho)\right\rangle_{\rho}=\kappa_{n}\left\langle\rho^{1 / 2} f(\rho), w_{n}(\rho)\right\rangle_{\rho}
$$

which leads to an analytical solution for the inverse source problem:

$$
\rho^{1 / 2} f(\rho)=\sum_{n=1}^{\infty} \kappa_{n}^{-1}\left\langle\rho^{1 / 2} g(\rho), w_{n}(\rho)\right\rangle_{\rho} w_{n}(\rho)
$$

Upon computing $\rho^{1 / 2} f(\rho)$, the radiogenic source term $f(\rho)$ can be obtained by multiplying $\rho^{-1 / 2}$.

Remark 2.1. Due to the singularity of the function $\rho^{-1 / 2}$, the radiogenic source function $f(\rho)$ is unable to be reconstructed at $\rho=0$ from the reconstructed function $\rho^{1 / 2} f(\rho)$. Fortunately, more attention is paid to the radiogenic source function at the edge for the stopping distance, i.e., when $\rho$ is close to $R$. In practice, the direct evaluation of $\rho^{-1 / 2}$ at $\rho=0$ will be unnecessary and thus can be avoided.

The analytical solution (2.11) involves the data function $g(\rho)$ and the source function $f(\rho)$ with a factor $\rho^{1 / 2}$. From the above discussion, we can reformulate the inverse source problem: To reconstruct the source function $f_{\rho}(\rho)$ from the given data function $g_{\rho}(\rho)$, where

$$
f_{\rho}(\rho)=\rho^{1 / 2} f(\rho) \quad \text { and } \quad g_{\rho}(\rho)=\rho^{1 / 2} g(\rho) .
$$


Since there may be some measurement errors in the data function $g_{\rho}$, the solution has to be reconstructed from noisy data $g_{\rho}^{\delta}$, which is assumed to satisfy

$$
\left\|g_{\rho}-g_{\rho}^{\delta}\right\|_{\rho} \leq \delta
$$

Here $\delta>0$ represents the noise level, and both $g_{\rho}$ and $g_{\rho}^{\delta}$ are assumed to be functions in $L^{2}([0, R], \rho)$. To derive the error estimates, it is necessary to assume certain regularity of the exact source function. Here we assume that there exists an a priori estimate for the source function $f_{\rho}$, i.e.,

$$
\left\|f_{\rho}\right\|_{\rho, p}<\infty, \text { for } p>0,
$$

where the norm is defined in terms of the eigenfunctions

$$
\left\|f_{\rho}\right\|_{\rho, p}=\left\|\sum_{n=1}^{\infty}\left(1+n^{2}\right)^{p / 2}\left\langle f_{\rho}, w_{n}\right\rangle_{\rho} w_{n}\right\|_{\rho}
$$

It is easy to check $\left\|f_{\rho}\right\|_{\rho, 0}=\left\|f_{\rho}\right\|_{\rho}$. This norm is equivalent to what we defined at the beginning of this section, but it will be more convenient to study the derivatives.

To study the ill-posed nature of the inverse problem, it is sufficient to investigate the decay property of the eigenvalues. Recall the lower and upper bounds for the diffusivity $a_{0}$ and $a_{1}$, respectively. Simple calculation yields the upper bound

$$
\kappa_{n} \leq \int_{0}^{T} \exp \left[-a_{0} \lambda_{n}(T-\tau)\right] d \tau \leq R^{2} /\left(a_{0} \pi^{2} n^{2}\right) \rightarrow 0 \quad \text { as } n \rightarrow \infty,
$$

and the lower bound

$$
\kappa_{n} \geq \int_{0}^{T} \exp \left[-a_{1} \lambda_{n}(T-\tau)\right] d \tau \geq R^{2} /\left(2 a_{1} \pi^{2} n^{2}\right) \rightarrow 0 \quad \text { as } n \rightarrow \infty .
$$

Since $f_{\rho}$ is in $L^{2}([0, R], \rho)$, the norm of $f_{\rho}$ in (2.13) implies the coefficient $\left\langle f_{\rho}, w_{n}\right\rangle_{\rho}=$ $\kappa_{n}^{-1}\left\langle g_{\rho}, w_{n}\right\rangle_{\rho}$ with respect to the eigenfunction $w_{n}$ decay fast enough relative to the eigenvalue $\kappa_{n}$. But such a decay is not likely to occur for the noisy data $g_{\rho}^{\delta}$ : error components which correspond to large eigenvalue are under control; error components which correspond to small eigenvalues $\kappa_{n}$ are amplified by the factors $\kappa_{n}^{-1}$, so that data errors of a fixed size can be amplified arbitrarily much, namely by the factors $\kappa_{n}^{-1}$, which increase without bound. On the other hand, due to the quadratic decay of the eigenvalues, the inverse problem is mildly ill-posed and good numerically results can still be expected after applying some regularization techniques.

\section{Problem regularization}

To obtain stable solution, we consider two classical regularization methods and derive the error estimates with convergence results. 


\subsection{Tikhonov regularization}

Define an operator $K: f_{\rho} \rightarrow g_{\rho}$, then the inverse source problem can be rewritten as the following operator equation:

$$
K f_{\rho}(\rho)=g_{\rho}(\rho), \quad 0<\rho<R
$$

Using (2.10), it holds

$$
K f_{\rho}(\rho)=\sum_{n=1}^{\infty} \kappa_{n}\left\langle f_{\rho}(\rho), w_{n}(\rho)\right\rangle_{\rho} w_{n}(\rho)
$$

Consequently, $K$ is a linear self-adjoint compact operator with eigenvalues $\kappa_{n}$ and eigenfunctions $w_{n}(\rho)$. For noisy data $g_{\rho}^{\delta}$, the Tikhonov regularization is to seek a function $f_{\rho}^{\delta, \alpha}$, which minimizes the cost functional

$$
M_{\alpha}(u)=\left\|K u-g_{\rho}^{\delta}\right\|_{\rho}^{2}+\alpha\|u\|_{\rho}^{2},
$$

where $\alpha>0$ is known as the regularization parameter.

Lemma 3.1. There exists a unique solution to the minimization problem (3.3) and the solution is given by

$$
f_{\rho}^{\delta, \alpha}=\sum_{n=1}^{\infty} \frac{\kappa_{n}}{\kappa_{n}^{2}+\alpha}\left\langle g_{\rho}^{\delta}, w_{n}\right\rangle_{\rho} w_{n} .
$$

Proof. Denote by $K^{*}$ the adjoint operator of $K$. It follows from [7] that the Tikhonov functional has a unique minimum $f_{\rho}^{\delta, \alpha} \in L^{2}([0, R], \rho)$, and $f_{\rho}^{\delta, \alpha}$ is the unique solution of the normal equation

$$
K^{*} K f_{\rho}^{\delta, \alpha}+\alpha f_{\rho}^{\delta, \alpha}=K^{*} g_{\rho}^{\delta}
$$

Since $K$ is a self-adjoint operator, i.e., $K^{*}=K$, combining (3.2) and (3.5) yields

$$
\sum_{n=1}^{\infty}\left(\kappa_{n}^{2}+\alpha\right)\left\langle f_{\rho}^{\delta, \alpha}, w_{n}\right\rangle_{\rho} w_{n}=\sum_{n=1}^{\infty} \kappa_{n}\left\langle g_{\rho}^{\delta}, w_{n}\right\rangle_{\rho} w_{n} .
$$

Thus we have

$$
\left(\kappa_{n}^{2}+\alpha\right)\left\langle f_{\rho}^{\delta, \alpha}, w_{n}\right\rangle_{\rho}=\kappa_{n}\left\langle g_{\rho}^{\delta}, w_{n}\right\rangle_{\rho}
$$

which gives

$$
\left\langle f_{\rho}^{\delta, \alpha}, w_{n}\right\rangle_{\rho}=\frac{\kappa_{n}}{\kappa_{n}^{2}+\alpha}\left\langle g_{\rho}^{\delta}, w_{n}\right\rangle_{\rho}
$$

Therefore it holds

$$
f_{\rho}^{\delta, \alpha}=\sum_{n=1}^{\infty}\left\langle f_{\rho}^{\delta, \alpha}, w_{n}\right\rangle_{\rho} w_{n}=\sum_{n=1}^{\infty} \frac{\kappa_{n}}{\kappa_{n}^{2}+\alpha}\left\langle g_{\rho}^{\delta}, w_{n}\right\rangle_{\rho} w_{n} .
$$

The proof is complete. 
The solution (3.4) is called the Tikhonov approximation of the exact solution (2.11). A comparison with (2.11) clearly shows the stabilization: errors in $\left\langle g_{\rho}^{\delta}, w_{n}\right\rangle_{\rho}$ are not propagate with the factors $\kappa_{n}^{-1}$, but only with the factors $\kappa_{n} /\left(\kappa_{n}^{2}+\alpha\right)$ into the result; these factors stay bounded as $n \rightarrow \infty$.

In order to prove the stability estimates for the regularized solution, we need the following elementary results.

Lemma 3.2. It holds

$$
\left(1-\frac{x}{4}\right)\left(\frac{x}{4-x}\right)^{x / 4} \leq \frac{1}{2}, \text { for } 0<x<4
$$

Proof. Define a function

$$
h_{2}(x)=\left(1-\frac{x}{4}\right)\left(\frac{x}{4-x}\right)^{x / 4}, \quad 0<x<4 .
$$

Under the change of variable $y=x / 4$, it becomes

$$
h_{2}(y)=(1-y)\left(\frac{y}{1-y}\right)^{y}, \quad 0<y<1
$$

It is easy to see that $h_{2}(y)$ is a positive function for $0<y<1$. Taking the logarithm on both sides yields

$$
\ln h_{2}(y)=\ln (1-y)+y \ln y-y \ln (1-y) .
$$

Taking the derivative with respect to $y$, we have

$$
\frac{h_{2}^{\prime}(y)}{h_{2}(y)}=\ln y-\ln (1-y),
$$

which yields

$$
h_{2}^{\prime}(1 / 2)=0 .
$$

Since the logarithmic function is a monotonely increasing function, the function $\ln h_{2}$ and thus the function $h_{2}$ reaches its maximum at $y=1 / 2$. Therefore, after changing back to the variable $x$, the function $h_{2}$ reaches its maximum at $x=2$ and the maximum is $h_{2}(2)=1 / 2$.

Lemma 3.3. Given $\alpha>0$ and $p>0$, it holds for all $n$

$$
\frac{\alpha}{\kappa_{n}^{2}+\alpha}\left(1+n^{2}\right)^{-p / 2} \leq c_{p} \alpha^{p / 4},
$$

where the coefficient $c_{p}$ is

$$
c_{p}= \begin{cases}c^{-p / 2}, & \text { for } p<4 \\ c^{-2}, & \text { for } p \geq 4\end{cases}
$$


Here the constant $c$ is

$$
c=\frac{\left[1-\exp \left(-a_{1} \lambda_{1} T\right)\right] R^{2}}{\pi^{2} a_{1}} .
$$

Proof. Recall $a_{1}$ is the upper bound of the diffusivity. Simple calculation yields the lower bounds for the eigenvalues

$$
\kappa_{n} \geq \int_{0}^{T} \exp \left[-a_{1} \lambda_{n}(T-\tau)\right] d \tau=\frac{1-\exp \left(-a_{1} \lambda_{1} T\right)}{a_{1} \lambda_{n}}=\frac{\left[1-\exp \left(-a_{1} \lambda_{1} T\right)\right] R^{2}}{\pi^{2} a_{1} n^{2}}=\frac{c}{n^{2}} .
$$

Using the lower bounds for the eigenvalues, we get

$$
\frac{\alpha}{\kappa_{n}^{2}+\alpha}\left(1+n^{2}\right)^{-p / 2} \leq \frac{\alpha n^{4-p}}{c^{2}+\alpha n^{4}}
$$

First consider the case $0<p<4$. Let

$$
h_{3}(x)=\frac{\alpha x^{4-p}}{c^{2}+\alpha x^{4}}, \quad \text { for } x>0 .
$$

By investigating the first and second derives of the function $h_{3}$, we can check that it reaches the maximum at the unique point $x=\left[(4-p) c^{2} / \alpha p\right]^{1 / 4}$, and the maximum is

$$
\left(1-\frac{p}{4}\right)\left[\frac{p}{c^{2}(4-p)}\right]^{p / 4} \alpha^{p / 4}
$$

It follows from Lemma 3.2 that

$$
h_{3}(x) \leq\left(1-\frac{p}{4}\right)\left[\frac{p}{c^{2}(4-p)}\right]^{p / 4} \alpha^{p / 4} \leq c^{-p / 2} \alpha^{p / 4}, \text { for } x>0 .
$$

Next consider the case $p \geq 4$. Let

$$
h_{4}(x)=\frac{\alpha}{\left(c^{2}+\alpha x^{4}\right) x^{p-4}}, \quad \text { for } x \geq 1
$$

Clearly we have

$$
h_{4}(x) \leq \frac{\alpha}{c^{2}+\alpha x^{4}} \leq c^{-2} \alpha, \text { for } x \geq 1 .
$$

The proof is complete by combining the above estimates.

Theorem 3.1. Let $f_{\rho}$ be the exact radiogenic source and $f_{\rho}^{\delta, \alpha}$ be the regularized approximation. It holds the stability estimate

$$
\left\|f_{\rho}-f_{\rho}^{\delta, \alpha}\right\|_{\rho} \leq\left(c_{p}\left\|f_{\rho}\right\|_{\rho, p}\right) \alpha^{p / 4}+\frac{\delta}{2 \sqrt{\alpha}} .
$$


Proof. It follows from the eigenfunction expansions of the exact solution (2.11) and the regularized solution (3.4) that we have

$$
\begin{aligned}
\left\|f_{\rho}-f_{\rho}^{\delta, \alpha}\right\|_{\rho} & =\left\|\sum_{n=1}^{\infty} \kappa_{n}^{-1}\left\langle g_{\rho}, w_{n}\right\rangle_{\rho} w_{n}-\sum_{n=1}^{\infty} \frac{\kappa_{n}}{\kappa_{n}^{2}+\alpha}\left\langle g_{\rho}^{\delta}, w_{n}\right\rangle_{\rho} w_{n}\right\|_{\rho} \\
& \leq\left\|\sum_{n=1}^{\infty} \frac{\alpha}{\kappa_{n}^{2}+\alpha} \kappa_{n}^{-1}\left\langle g_{\rho}, w_{n}\right\rangle_{\rho} w_{n}\right\|_{\rho}+\left\|\sum_{n=1}^{\infty} \frac{\kappa_{n}}{\kappa_{n}^{2}+\alpha}\left\langle g_{\rho}-g_{\rho}^{\delta}, w_{n}\right\rangle_{\rho} w_{n}\right\|_{\rho} .
\end{aligned}
$$

Simple calculation yields

$$
\left\|f_{\rho}-f_{\rho}^{\delta, \alpha}\right\|_{\rho} \leq\left\|\sum_{n=1}^{\infty} \frac{\alpha\left(1+n^{2}\right)^{-p / 2}}{\kappa_{n}^{2}+\alpha}\left(1+n^{2}\right)^{p / 2} \kappa_{n}^{-1}\left\langle g_{\rho}, w_{n}\right\rangle_{\rho} w_{n}\right\|_{\rho}+\frac{1}{2 \sqrt{\alpha}}\left\|g_{\rho}-g_{\rho}^{\delta}\right\|_{\rho} .
$$

An application of Lemma 3.3 gives

$$
\left\|f_{\rho}-f_{\rho}^{\delta, \alpha}\right\|_{\rho} \leq\left(c_{p}\left\|f_{\rho}\right\|_{\rho, p}\right) \alpha^{p / 4}+\frac{\delta}{2 \sqrt{\alpha}}
$$

which completes the proof.

Following the stability estimate (3.6), we observe if the regularization parameter $\alpha$ becomes too small, the total error increases due to the error term $\delta /(2 \sqrt{\alpha})$, the propagated data error. Certainly, if $\alpha$ is too large, then the approximation error becomes too large. There is an optimal regularization parameter $\alpha$, which can not be explicitly computed, since it depends on unavailable information about the exact source function. However, one can estimate the asymptotic behaviour of the total error if $\alpha$ is chosen as a power of $\delta$.

Remark 3.1. If the regularization parameter is chosen as $\alpha=\delta^{\frac{4}{p+2}}$, then the stability estimate (3.6) reduces to

$$
\left\|f_{\rho}-f_{\rho}^{\delta, \alpha}\right\|_{\rho} \leq\left(c_{p}\left\|f_{\rho}\right\|_{\rho, p}+\frac{1}{2}\right) \delta^{\frac{p}{p+2}}
$$

\subsection{Spectral cut-off regularization}

Besides the Tikhonov regularization method, another commonly used regularization method is called the spectral cut-off or the truncated singular value decomposition. Choose a positive number $\beta>0$, the spectral cut-off method gives a regularized solution

$$
f_{\rho}^{\delta, \beta}=\sum_{\kappa_{n} \geq \beta} \kappa_{n}^{-1}\left\langle g_{\rho}^{\delta}, w_{n}\right\rangle_{\rho} w_{n}
$$

where $\beta$ is also known as the regularization parameter.

Similar to the stability estimate of the Tikhonov regularization method, the stability estimate of the spectral cut-off is also composed of two error terms, one for the approximation error, the other one for the propagation of the data error. 
Theorem 3.2. Let $f_{\rho}$ be the exact radiogenic source and $f_{\rho}^{\delta}$ be the regularized approximation. It holds the stability estimate

$$
\left\|f_{\rho}-f_{\rho}^{\delta, \beta}\right\|_{\rho} \leq\left(c^{-q}\left\|f_{\rho}\right\|_{\rho, p}\right) \beta^{q}+\frac{\delta}{\beta^{\prime}}
$$

where $q>0, p>0$ satisfying $p \geq 2 q$.

Proof. Following the eigenfunction expansion for the exact solution (2.11) and the regularized solution (3.8), we obtain

$$
\begin{aligned}
\left\|f_{\rho}-f_{\rho}^{\delta, \beta}\right\|_{\rho} & =\left\|\sum_{n=1}^{\infty} \kappa_{n}^{-1}\left\langle g_{\rho}, w_{n}\right\rangle_{\rho} w_{n}-\sum_{\kappa_{n} \geq \beta} \kappa_{n}^{-1}\left\langle g_{\rho}^{\delta}, w_{n}\right\rangle_{\rho} w_{n}\right\|_{\rho} \\
& \leq\left\|\sum_{n=1}^{\infty} \kappa_{n}^{-1}\left\langle g_{\rho}, w_{n}\right\rangle_{\rho} w_{n}-\sum_{\kappa_{n} \geq \beta} \kappa_{n}^{-1}\left\langle g_{\rho}, w_{n}\right\rangle_{\rho} w_{n}\right\|_{\rho}+\left\|\sum_{\kappa_{n} \geq \beta} \kappa_{n}^{-1}\left\langle g_{\rho}-g_{\rho}^{\delta}, w_{n}\right\rangle_{\rho} w_{n}\right\|_{\rho},
\end{aligned}
$$

which deduces to

$$
\left\|f_{\rho}-f_{\rho}^{\delta, \beta}\right\|_{\rho} \leq\left\|\sum_{\kappa_{n}<\beta} \kappa_{n}^{-1}\left\langle g_{\rho}, w_{n}\right\rangle_{\rho} w_{n}\right\|_{\rho}+\frac{1}{\beta}\left\|g_{\rho}-g_{\rho}^{\delta}\right\|_{\rho} .
$$

For any given $q>0$, we may obtain an estimate for the first term of the right hand side

$$
\begin{aligned}
\left\|\sum_{\kappa_{n}<\beta} \kappa_{n}^{-1}\left\langle g_{\rho}, w_{n}\right\rangle_{\rho} w_{n}\right\|_{\rho} & \leq\left\|\sum_{n=1}^{\infty}\left(\frac{\beta}{\kappa_{n}}\right)^{q} \kappa_{n}^{-1}\left\langle g_{\rho}, w_{n}\right\rangle_{\rho} w_{n}\right\|_{\rho} \\
& \leq \beta^{q}\left\|\sum_{n=1}^{\infty} \frac{\left(1+n^{2}\right)^{-p / 2}}{\kappa_{n}^{q}}\left(1+n^{2}\right)^{p / 2} \kappa_{n}^{-1}\left\langle g_{\rho}, w_{n}\right\rangle_{\rho} w_{n}\right\|_{\rho} .
\end{aligned}
$$

Recalling $\kappa_{n} \geq c / n^{2}$ and using the inequality $p \geq 2 q$, we get

$$
\left\|\sum_{\kappa_{n}<\beta} \kappa_{n}^{-1}\left\langle g_{\rho}, w_{n}\right\rangle_{\rho} w_{n}\right\|_{\rho} \leq\left(c^{-q}\left\|f_{\rho}\right\|_{\rho, p}\right) \beta^{q} .
$$

The proof is complete by combining the above estimates.

We may also estimate the asymptotic behaviour of the total error if $\beta$ is chosen as a power of $\delta$.

Remark 3.2. If the regularization parameter is chosen as $\beta=\delta^{\frac{1}{1+q}}$, then the stability estimate (3.9) reduces to

$$
\left\|f_{\rho}-f_{\rho}^{\delta, \beta}\right\|_{\rho} \leq\left(c^{-q}\left\|f_{\rho}\right\|_{\rho, p}+1\right) \delta^{\frac{q}{1+q}}
$$

where $p \geq 2 q>0$. Since $q /(1+q)$ is an increasing function of $q$, for a given $p$, the maximum order of convergence is $\frac{p}{2+p}$ when $q=p / 2$. 


\section{Numerical experiments}

In this section, we discuss the algorithmic implementation for the direct and inverse radiogenic source problems, and present three numerical examples to illustrate the validity and effectiveness of the proposed method.

\subsection{Direct problem}

Ignoring the radial symmetry of the production-diffusion model equation (1.1), we consider the helium model equation in the three-dimensional space with Cartesian coordinate:

$$
\frac{\partial u(t, \mathbf{x})}{\partial t}=a(t) \Delta u(t, \mathbf{x})+f(\mathbf{x}) \quad \text { in }(0, T) \times B,
$$

together with homogeneous boundary condition

$$
u(t, \mathbf{x})=0 \quad \text { on }(0, T) \times S,
$$

and the homogeneous initial condition

$$
u(0, \mathbf{x})=0 \quad \text { in } B,
$$

where $B$ is the unit ball and $S$ is the unit sphere. Assume that $a \in L^{\infty}([0, T])$ and $f \in L^{2}(B)$. It follows from Ladyženskaja et al. [16] that there is a unique weak solution $u \in C([0, T]) \times$ $H_{0}(B)$ for the above model equations.

To generate the data $u(t, \mathbf{x})$ at the final time $T$, we adopt the finite element method which is to solve the variational problem: To find $u(t, \mathbf{x})$ such that

$$
\frac{d}{d t} \int_{B} u(t, \mathbf{x}) \varphi(\mathbf{x}) d \mathbf{x}+a(t) \int_{B} \nabla u(t, \mathbf{x}) \cdot \nabla \varphi(\mathbf{x}) d \mathbf{x}=\int_{B} f(\mathbf{x}) \varphi(\mathbf{x}) d \mathbf{x}
$$

for all $\varphi(\mathbf{x}) \in H^{1}(B)$.

To create the mesh for the finite element method, we employ a simple and effective mesh generator in Matlab by Persson and Strang [17]. In the spatial domain, we choose the continuous piecewise linear polynomial for the tetrahedra, and the backward Euler method is used in the temporal domain for the variational problem. In the presented numerical examples, using meshsize 0.05 , the unit ball is divided into 190,976 tetrahedra elements with 34,049 nodes, and the time interval $[0,1]$ is equally divided into 400 subintervals.

Upon computing the data $u(T, \mathbf{x})$, we shall project it onto the one-dimensional function $u(T, \rho)=g(\rho)$ where $\rho=|\mathbf{x}|$. Due to the unstructured mesh, the projected data $u(T, \rho)$ from $u(T, \mathbf{x})$ will not be equally spaced with respect to the spatial variable $\rho$. We construct a smooth curve $g(\rho)$ by using the natural cubic spline interpolation formula based on the computed discrete data $u(T,|\mathbf{x}|)$. 
For simplicity, we assume that the diffusivity $a(t)$ is a constant. The eigensystem can be explicitly computed for the constant diffusivity. More specifically, simple calculations yield the eigenvalues

$$
\kappa_{n}=\int_{0}^{T} \exp \left[-\lambda_{n} \int_{\tau}^{T} a\right] d \tau=\left[1-\exp \left(-a \lambda_{n} T\right)\right] /\left(a \lambda_{n}\right),
$$

and the eigenfunctions

$$
w_{n}(\rho)=\frac{\sqrt{2}}{J_{3 / 2}\left(\mu_{n}\right)} y_{n}(\rho)=(-1)^{n-1} \sqrt{\frac{2}{\rho}} \sin (n \pi \rho) .
$$

Substituting the eigenfunctions gives a formula to compute the coefficients

$$
\left\langle\rho^{1 / 2} g(\rho), w_{n}(\rho)\right\rangle_{\rho}=(-1)^{n-1} \sqrt{2} \int_{0}^{1} \rho g(\rho) \sin (n \pi \rho) d \rho .
$$

Note that the right hand side is essentially the sine transform of the function $\rho g(\rho)$, which can be efficiently implemented by using a version of the fast Fourier transform for real functions [18].

Based on the explicit expressions for the eigensystems, we may deduce the final solution representation for the Tikhonov regularization

$$
f^{\delta, \alpha}=\frac{2}{\rho} \sum_{n=1}^{\infty} \frac{\kappa_{n}}{\kappa_{n}^{2}+\alpha}\left[\int_{0}^{1} \rho g^{\delta}(\rho) \sin (n \pi \rho) d \rho\right] \sin (n \pi \rho),
$$

and for the spectral cut-off

$$
f^{\delta, \beta}=\frac{2}{\rho} \sum_{\kappa_{n} \geq \beta} \kappa_{n}^{-1}\left[\int_{0}^{1} \rho g^{\delta}(\rho) \sin (n \pi \rho) d \rho\right] \sin (n \pi \rho),
$$

where

$$
g^{\delta}(\rho)=g(\rho) \cdot[1+\delta \operatorname{rand}(\rho)]
$$

Here rand gives uniformly distributed random number in the interval $[-1,1]$.

\subsection{Inverse problem}

Numerical experiments have been done for wide classes of functions. We present three examples to illustrate the performance of the proposed methods. The solutions display similar patterns for the Tikhonov regularization method and the spectral cut-off method. Thus we will only show the results computed from either the Tikhonov regularization or the spectral cut-off. 


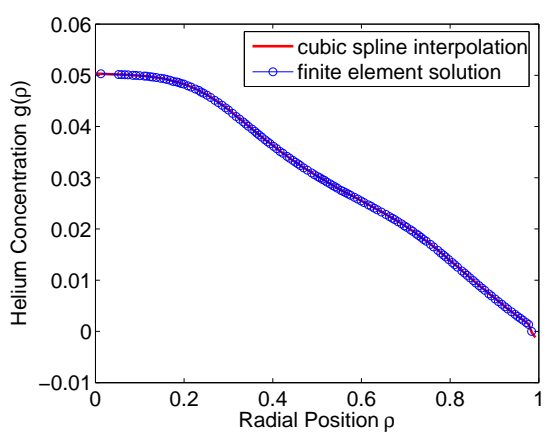

Figure 1: The unperturbed data function $g(\rho)$ for Example 4.1.

Example 4.1. Reconstruct the radiogenic source

$$
f_{1}(\rho)=\frac{1}{3}\left(1-\rho^{2}\right) \exp [-\cos (4 \pi \rho)]
$$

over the interval $[0,1]$. This is a simple example as it is infinitely smooth function. Fig. 1 shows the unperturbed data function $g(\rho)$ computed from the finite element method and interpolated by using the natural cubic spline formula. Fig. 2 plots the reconstructed

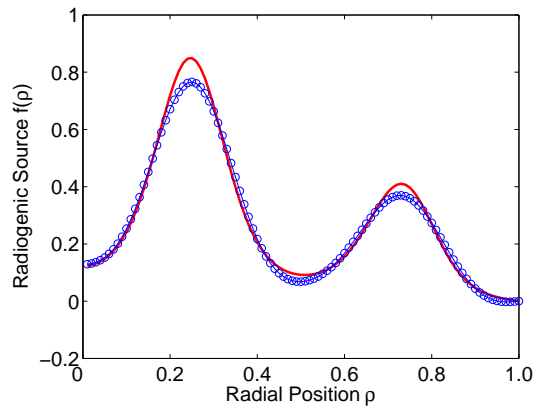

(a)

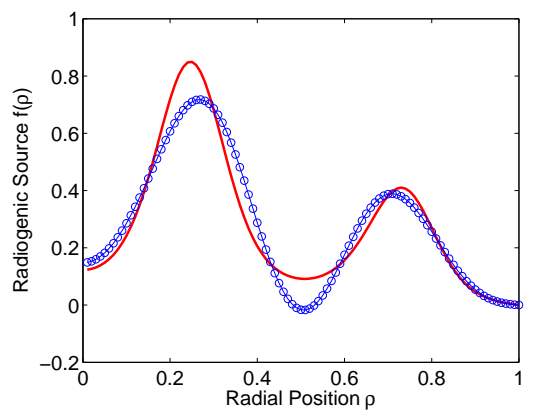

(c)

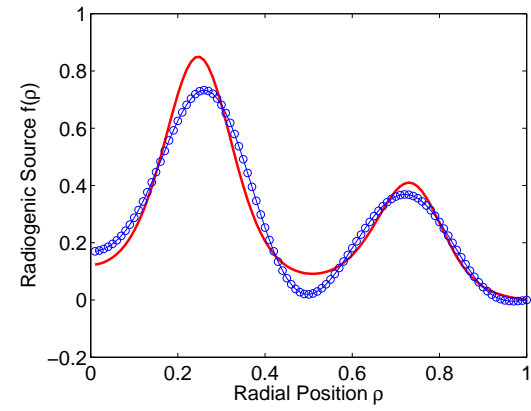

(b)

Figure 2: Reconstructed source function against exact one for Example 4.1. (a) $\delta=1 \%, N=9$; (b) $\delta=3 \%, N=8$; (c) $\delta=5 \%, N=7$. 
source functions from the spectral cut-off method against the exact one. The choice of the regularization parameter $\beta$ is equivalent to the choice of number of the terms $N$ in the eigenfunction expansion in (4.2). It can be seen from Fig. 2 that the number of terms $N$ should decrease, equivalently increase the regularization parameter $\beta$, as the noise level $\delta$ increase. As expected, the results are more accurate at the right end side that at the left end side.

Example 4.2. Reconstruct the radiogenic source

$$
f_{2}(\rho)=0.5[1+\cos (7 \pi \rho)]
$$

over the interval $[0,1]$. This is a highly oscillatory function and more terms of expansion is desired in order to capture the high Fourier modes of the function. Fig. 3 shows the unperturbed data function $g(\rho)$ computed from the finite element method and interpolated by using the natural cubic spline formula. Fig. 4 plots the reconstructed source functions from the Tikhonov regularization method against the exact one. The regularization parameter is chosen as $\alpha=10^{-6}$. Though the factors $\kappa_{n} /\left(\kappa_{n}^{2}+\alpha\right)$ stay bounded as $n \rightarrow \infty$, only finitely many terms are computed in practice. So Fig. 4 presents the results with a combination of the Tikhonov regularization method and the spectral cut-off method. Again, it can be seen from Fig. 4 that the number of terms $N$ should decrease as the noise level $\delta$ increase for a fixed regularization parameter $\alpha$. It yields the similar results if we increase the regularization parameter $\alpha$ while keeping $N$ as a constant.

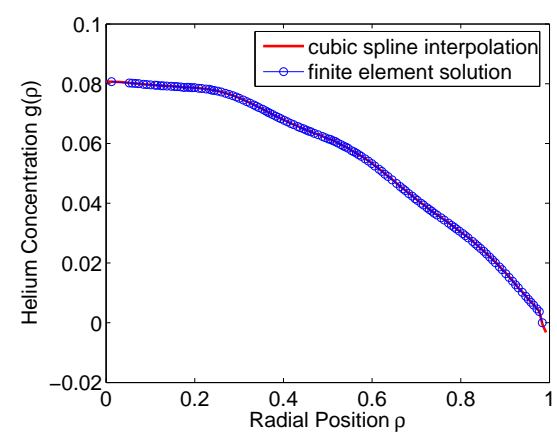

Figure 3: The unperturbed data function $g(\rho)$ for Example 4.2.

Example 4.3. Reconstruct the radiogenic source

$$
f_{3}(\rho)= \begin{cases}1, & \text { for } 0<\rho<0.5 \\ 16(1-\rho)^{4}, & \text { for } 0.5<\rho<1\end{cases}
$$

over the interval $[0,1]$. This is a more difficult example as the function is not smooth on the point $\rho=0.5$. Fig. 5 shows the unperturbed data function $g(\rho)$ computed from the 


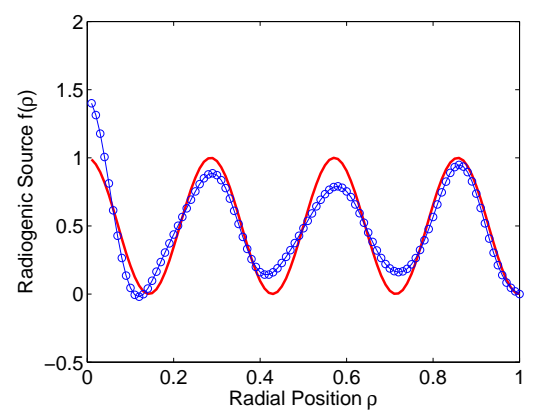

(a)

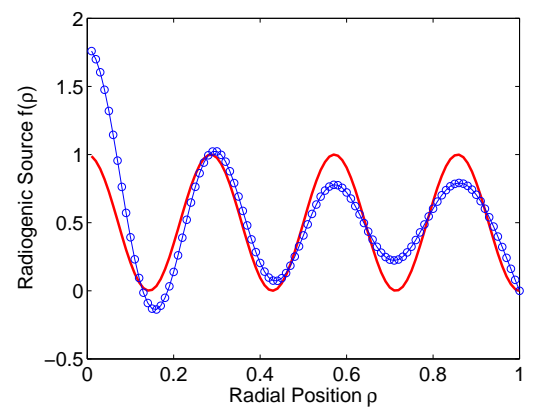

(c)

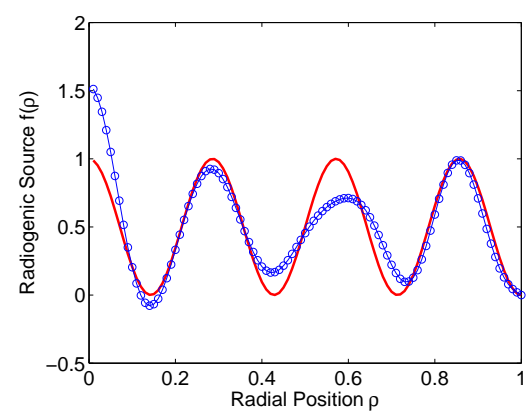

(b)

Figure 4: Reconstructed source function against exact one for Example 4.2. (a) $\delta=1 \%, \alpha=10^{-6}, N=14$; (b) $\delta=3 \%$, $\alpha=10^{-6}, N=12$; (c) $\delta=5 \%, \alpha=10^{-6}$, $N=8$.

finite element method and interpolated by using the natural cubic spline formula. Fig. 6 plots the reconstructed source functions from the combination of the Tikhonov and spectral cut-off method against the exact one. Due to the low regularity of the exact source function, it follows form the stability estimates that larger regularization parameters are expected to obtain a stable solution. As the noise level $\delta$ increases from $1 \%$ to $5 \%$, the regularization parameters vary from $\alpha=10^{-5}, N=6$ to $\alpha=10^{-5}, N=5$. More accurate results are still obtained toward the right end side of the interval.

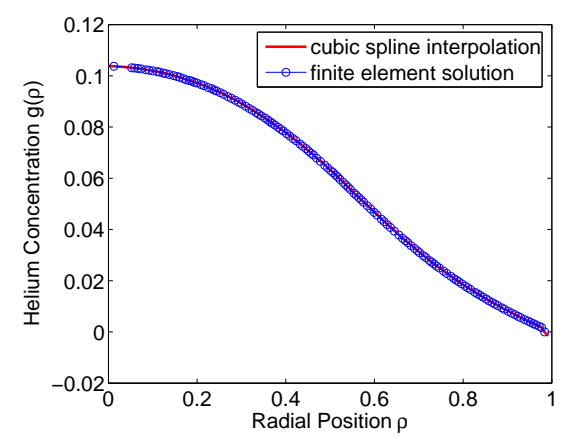

Figure 5: The unperturbed data function $g(\rho)$ for Example 4.3. 


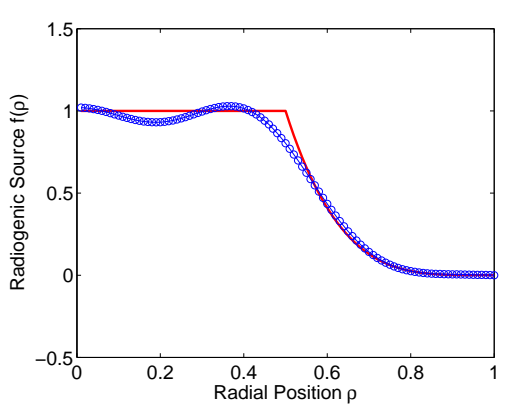

(a)

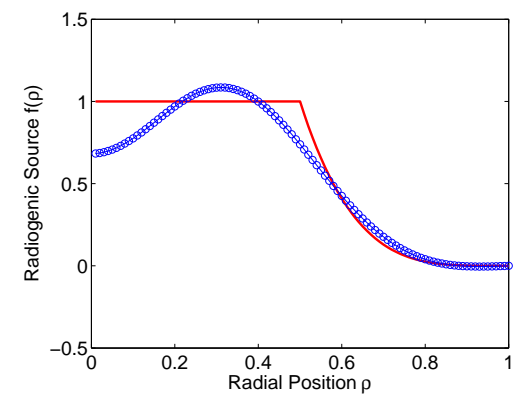

(c)

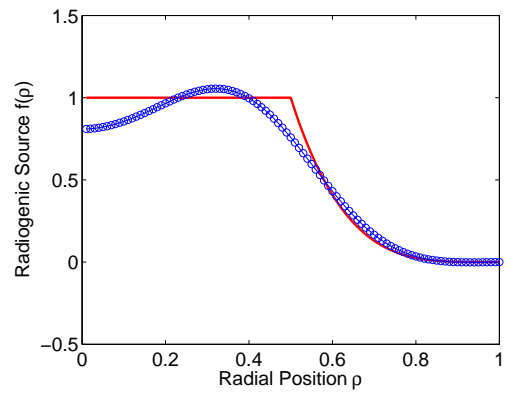

(b)

Figure 6: Reconstructed source function against exact one for Example 4.3. (a) $\delta=1 \%, \alpha=10^{-6}, N=6$; (b) $\delta=3 \%$, $\alpha=10^{-5}, N=5$; (c) $\delta=5 \%, \alpha=10^{-5}$, $N=5$.

\section{Concluding remarks}

In thermochronology, it is important to understand spatial variations in radiogenic sources in a sample as they can cause substantial spatial fractionation of the parent/daughter ratio in systems (such as rocks). In this paper, the prediction of a variable source term has been formulated as an inverse radiogenic source problem. Based on the radial geometry, an analytical solution has been deduced in terms of the eigenfunction expansion. Both the Tikhonov regularization method and the spectral cutoff regularization method have been considered to obtain the stable solution. Numerical results indicate that the proposed methods are efficient, accurate, and stable to compute the approximation of the unknown radiogenic source.

We conclude the paper by some general remarks about future directions along this line of research. The choice of a suitable value of the regularization parameter is crucial for the accuracy and the stability of the numerical solution, and is still under intensive investigation [7]. We are also investigating the inverse radiogenic source problem for general geometry where the eigenfunction expansion will not be available any more.

\section{Acknowledgments}

The research of GB was supported in part by the NSF grants DMS-0908325, CCF-0830161, EAR-0724527, and DMS-0968360, the ONR grant N00014-12-1-0319, a Key Project of the 
Major Research Plan of NSFC (No. 91130004), and a special research grant from Zhejiang University. The research of TE was supported in part by the NSF grant EAR-0724656 and German Science Foundation grant DFG-EH 328/1-1. The research of PL was supported in part by the NSF grants EAR-0724656, DMS-0914595, and DMS-1042958.

\section{References}

[1] G. Bao, Y. Dou, T. Ehlers, P. Li, Y. Wang, and Z. Xu, Quantifying tectonic and geomorphic interpretations of thermochronometer data: the reconstruction of mountain surface, Commun. Comput. Phys., 9 (2011), 129-146.

[2] I. Bushuyev, Global uniqueness for inverse parabolic problems with final observation, Inverse Problems, 11 (1995), Lll-L16.

[3] J. Cannon, The One-Dimensional Heat Equation, Cambridge University Press, 1984.

[4] J. Cannon and S. Pérez-Esteva, Uniqueness and stability of 3D heat sources, Inverse Problems, 7 (1991), 57-62.

[5] W. Cheng, L. Zhao, C. Fu, Source term identification for an axisymmetric inverse heat conducting problem, Comput. Math. Appl., 59 (2010), 142-148.

[6] M. Choulli and M. Yamamoto, Conditional stability in determing a heat source, J. Inverse Ill-posed Probl., 12 (2004), 233-243.

[7] H. Engl, M. Hanke, and A. Neubauer, Regularization of Inverse Problems, Dordrecht: Kluwer, 1996.

[8] A. Farcas and D. Lesnic, The boundary element method for the determination of a heat source dependent on one variable, Journal of Engineering Mathematics, 54 (2006), 375-388.

[9] K. Farley, R. Wolf, and L. Silver, The effects of long alpha-stopping distance on (U-Th)/He ages, Geochim. Cosmochim. Acta, 60 (1996), 4223-4230.

[10] F. Hettlich and W. Rundell, Identification of a discontinuous source in the heat equation, Inverse Problems, 17 (2001), 1465-1482.

[11] V. Isakov, Inverse Source Problems, American Mathematical Society, Providence, RI, 1990.

[12] V. Isakov, Inverse parabolic problems with the final overdetermination, Comm. Pure Appl. Math., 44 (1991), 185-209.

[13] V. Isakov, Inverse Problems for Partial Differential Equations, Springer-Verlag, New York, 1998.

[14] T. Johansson and D. Lesnic, A variational method for identifying a spacewise-dependent heat source, IMA J. Appl. Math., 72 (2007), 748-760.

[15] A. Kirsch, An Introduction to the Mathematical Theory of Inverse Problem, Springer-Verlag, New York, 1996.

[16] O. A. Ladyženskaja, V. A. Solonnikov, and N. N. Ural'ceva, Linear and Quasilinear Equations of Parabolic Type, Translations of Mathematical Monographs, vol. 23, American Mathematical Society, Providence, RI, 1967.

[17] P. Persson and G. Strang, A simple mesh generator in Matlab, SIAM Rev., 46 (2004), 329-345.

[18] W. Press, S. Teukolsky, W. Vetterling, and B. Flannery, Numerical Recipes in Fortran 90: The Art of Parallel Scientific Computing, 2nd ed., Cambridge University Press, 1996.

[19] K. Sakamoto and M. Yamamoto, Inverse heat source problem from time distributing overdetermination, Applicable Analysis, 88 (2009), 735-748.

[20] D. Shuster and K. Farley, ${ }^{4} \mathrm{He} /{ }^{3} \mathrm{He}$ thermochronometry, Earth Planet. Sci. Lett., 217 (2003), $1-17$. 
[21] G. Watson, A Treatise on the Theory of Bessel Functions, Cambridge University Press, 1995

[22] R. Wolf, K. Farley, and D. Kass, Modeling of the temperature sensitivity of the apatite (UTh)/He thermochrometer, Chem. Geol., 148 (1998), 105-114.

[23] R. Wolf, K. Farley, and L. Silver, Helium diffusion and low temperature thermochronometry of apatite, Geochim. Cosmochim. Acta, 60 (1996), 4231-4240.

[24] J. Xie and J. Zou, Numerical reconstruction of heat fluxes, SIAM J. Numer. Anal., 43 (2005), 1504-1535.

[25] M. Yamamoto and J. Zou, Simultaneous reconstruction of the initial temperature and heat radiative coefficient, Inverse Problems, 17 (2001), 1181-1202.

[26] L. Yan, C. Fu, and F. Dou, A computational method for identifying a spacewise-dependent heat source, Int. J. Numer. Meth. Biomed. Engng., 26 (2010), 597-608.

[27] Z. Yi and D. Murio, Source term identification in the 1-D IHCP, Computers Math. Applic., 47 (2004), 1921-1933.

[28] Z. Yi and D. Murio, Source term identification in the 2-D IHCP, Computers Math. Applic., 47 (2004), 1517-1533. 